\title{
Intérêt du dosage de la testostérone biodisponible dans les dysérections
}

\author{
J. Devignes*, W. Mahmoud**, J. Gúchot*, G. Arvis** et J. Giboudeau* \\ * Laboratoire de Biochimie-Hormonologie, Hôpital Saint-Antoine, 184, rue du Faubourg \\ Saint-Antoine 75571 Paris Cedex 12 ; ** Service d'Urologie et d'Andrologie, Hôpital Tenon, \\ 4, rue de la Chine, 75020 Paris, France.
}

\section{RESUME}

La testostérone biodisponible est-elle plus sensible que la testostérone totale pour la mise en évidence d'un hypogonadisme des patients atteints de dysfonction érectile ? Une diminution de la testostérone biodisponible ou testostérone non liée à la protéine de transport de l'hormone sexuelle (SHBG) et une augmentation de la SHBG dans le sérum à partir de l'âge moyen ont déjà été décrites. La SHBG sérique augmente dans les étiologies organiques des dysfonctions érectiles. Le but de ce travail est d'étudier le statut hormonal des sujet atteints de dysfonction érectile. La SHBG, la testostérone (T), la T biodisponible, l'hormone lutéinique (LH), l'hormone stimulante folliculaire (FSH), ont été dosées dans le sérum de 40 sujets atteints de dysfonction érectile. Ces 40 sujets ont été divisés en 4 groupes selon leur étiologie : psychogénique, vasculogénique, iatrogénique, et idiopathique. Dans le but de prendre en compte l'effet de l'âge, chaque groupe était comparé à un groupe témoin de sujets sains de même âge. La $T$ biodisponible est diminuée et la SHBG augmentée dans le groupe de sujets d'âge moyen $52 \pm 8$ ans par rapport au groupe de sujets jeunes d'âge moyen $28 \pm 3$ ans alors que la testostérone sérique n'était pas significative- ment différente dans les deux groupes. Dans le groupe d'étiologie vasculaire, la SHBG sérique était en moyenne plus élevée que dans le groupe témoin, mais de façon non significative. Dans le groupe de patients d'étiologies idiopathiques, la $T$ biodisponible était plus basse que dans le groupe témoin de même âge et sans augmentation de la SHBG sérique. Dans le groupe de patients d'étiologie psychogénique, la SHBG sérique était plus élevé que dans le groupe témoin de même âge, tandis que la testostérone totale restait similaire entre les deux groupes. Cette étude a permis d'étudier le statut androgénique de sujet atteints de dysfonction érectile selon leurs principales étiologies. L'étape suivante est d'étudier le succès d'une thérapie hormonale chez ces patients lorsqu'un hypogonadisme périphérique apparaît.

Mots-clefs : dysfonction érectile, hypogonadisme, testostérone biodisponible.

\section{INTRODUCTION}

Les dysfonctions érectiles affectent un grand nombre d'hommes. C'est une cause fréquente de consultation, particulièrement de la part des hommes à partir de la cinquantaine. L'enquête étiologique n'est pas si aisée car les causes sont souvent multiples, cependant le traitement doit être soi- 
gneusement adapté. Des recherches fondamentales sur les mécanismes physiologiques, biochimiques, physiopathologiques et les réponses aux traitements des différentes formes de ces dysfonctions apparaissent nécessaires.

L'effet spécifique des hormones (spécialement des androgènes) sur les fonctions sexuelles, la détermination de la fréquence des causes endocriniennes (hypogonadisme et hyperprolactinémie) et l'efficacité des thérapeutiques hormonales appropriés doivent être évaluées.

La testostérone totale sérique est-elle moins sensible que la testostérone biodisponible pour évaluer l'hypogonadisme chez l'homme atteint de dysfonction érectile ? Ce point est important pour déterminer quels sont les patients bon répondeurs à une thérapeutique hormonale.

A notre connaissance, seulement une étude antérieure sur le statut androgénique des patients atteints de dysfonction érectile secondaire [23] montre que la SHBG sérique augmente dans les étiologies psychogéniques et vasculaires et que des modifications du bilan hormonal androgénique sont constatées aussi bien dans des étiologies organiques qu'idiopathiques. Une augmentation du taux sérique de la SHBG et une diminution de la testostérone libre dans le sérum ont été rapportées dans les dysfonctions érectiles associées aux hypogonadismes secondaires [11]. Le taux sérique de SHBG augmente chez les patients diabétiques atteints de dysfonction érectile [10]. La testostérone libre sérique est significativement plus basse dans ce groupe que dans les groupes de patients diabétiques sans dysfonction érectile. Il en est de même de la testostérone totale. De même, la diminution de la testostérone totale et de sa fraction libre [7], une augmentation de la SHBG sérique [3] ont été mises en évidence dans un groupe de patients alcooliques atteints de dysfonction érectile. Dans l'hémochromatose, l'hypogonadisme lié à l'atteinte gonadique entraîne une augmentation de la SHBG sérique [4]. L'apparition d'une cirrhose du foie potentialise cet effet. Une augmentation de la SHBG sérique a été décrite dans un groupe de patients atteints d'hémochromatose et de dysfonction érectile [12], dans un groupe de patients insuffisants respiratoires et hypoxémiques, atteints de dysfonction érectile [1], un groupe de patients atteints de maladie coliaque [8], dans l'hyperthyroïdie et l'hyperprolactinémie [20]; les concentrations de la SHBG sériques sont aussi modifiées par des médicaments tels que les androgènes, les cestrogènes, et les hormones thyroïdiennes [20]. Dans un étude sur une longue période, 250 mg d'énanthate de testostérone permettaient le rétablissement d'une fonction érectile normale chez des patients atteints d'hémochromatose [12].

La testostérone sérique se lie en grande partie à la SHBG, à l'albumine, et un faible pourcentage est non lié. Elle constitue la testostérone libre ou fraction libre de la testostérone (FT). Autrefois, la testostérone libre était considérée comme la fraction biodisponible, mais des études récentes suggèrent que dans les conditions physiologiques, la testostérone biodisponible comprend la fraction libre de la testostérone et la fraction liée à l'albumine. La testostérone biodisponible présente une meilleure corrélation avec l'activité des androgènes que la testostérone totale ou sa fraction libre; et la testostérone biodisponible peut être estimée en déterminant la testostérone non liée à la SHBG (T biodisponible) [18]. La T biodisponible est plus basse chez les sujets âgés en comparaison des sujets jeunes $[5,6]$ et le changement a lieu à partir de la cinquantaine [18]. Au contraire, les mêmes auteurs, ont montré que FT et l'hormone lutéinique (LH) ont des concentrations comparables chez les sujets jeunes et chez les sujets âgés. D'autre part, les dysfonctions érectiles sont plus fréquentes chez les sujets âgés avec une fréquence d'un sur quatre $[16,19,21,22]$. Les modifications de 
la SHBG sérique sont probablement liées à une fréquence plus élevée des pathologies organiques chez les sujets âgés.

Dans ce travail nous avons étudié les variations de la testostérone sérique, de la testostérone biodisponible et leurs corrélations avec différentes étiologies de dysfonctions érectiles dans le but de définir plus clairement les patients à traiter avec des androgènes. Pour répondre à cette question, nous avons étudié la testostérone totale, T biodisponible, et la SHBG sérique dans des groupes de patients sans ou avec des signes de dysfonctions érectiles d'origine organique ou idiopathique. L'œstradiol (E2), les gonadotrophines, l'hormone stimulante du follicule (FSH) et la LH étaient aussi étudiées.

\section{MATERIEL ET METHODES}

Quarante patients (âge moyen $51 \pm 8$ années) ont été successivement inclus dans une étude prospective alors qu'ils venaient à l'hôpital pour un bilan de dysfonction érectile. Ils ont donné leur consentement pour la participation à cette étude. Les patients atteints de diabète, de pathologie thyroïdienne, d'hyperprolactinémie, et d'alcoolisme étaient systématiquement exclus de cette étude.

Les symptômes de dysfonction érectile allaient du simple défaut de maintien (dans 31 cas) à l'anérection complète (dans 9 cas).

Le diagnostic étiologique était établi par des praticiens spécialisés en andrologie et des psychiatres sur la base de l'histoire clinique et de l'activité sexuelle, d'un examen physique et d'une étude psychosociale, et de tests de laboratoires. Ces examens de laboratoires comprenaient les dosages de $\mathrm{T}, \mathrm{T}$ biodisponible, SHBG, LH, FSH, et de prolactine sérique. Eventuellement, selon l'orientation clinique, d'autres tests étaient pratiqués dans le but d'exclure une maladie systémique tels qu'une numération formule sanguine, une analyse d'urine, une créatini- ne sérique, les lipides sériques, une glycémie à jeun et post prandiale, et le dosage des hormones thyroïdiennes. Une pléthysmographie pénienne nocturne était pratiquée chez les patients qui se plaignaient d'une absence complète d'érection ou lorsqu'une étiologie psychogène était suspectée.

De même, en présence de signes cliniques d'atteinte vasculaire, d'autres tests étaient pratiqués. Dans le cas de suspicion d'artériopathie, une échographie avec ultrasonographie était pratiquée et dans le cas de suspicion de fuite veineuse caverneuse, une cavernométrie avec cavernographie était réalisée. Enfin, en cas de suspicion d'atteinte neurovégétative, un enregistrement neurophysiologique était fait.

Un groupe de 19 sujets sains sans aucune dysfonction érectile et sans aucune dysfonction gonadique ou d'hypoandrogénie étaient volontaires pour participer comme groupe contrôle à l'étude hormonale. Dans le but de prendre en compte l'effet de l'âge sur le statut gonadique, deux sous-groupes de contrôle selon l'âge, 10 hommes âgés de 23 à 32 ans $(28 \pm 3)$ et 9 hommes âgés de 43 à 69 ans ( $52 \pm 8$ ), étaient constitués.

\section{Les prélèvements de sang}

Les échantillons de sang étaient prélevés entre 8 heures et 10 heures. Les test hormonologiques étant différés : les sérums étaient, après centrifugation, fractionnés et stockés en plusieurs aliquots à $-20^{\circ} \mathrm{C}$ jusqu'à l'essai.

\section{Les tests hormonaux}

Des tests radioimmunologiques (RIA) sensibles et spécifiques étaient utilisés pour mesurer la concentration sérique de la testostérone totale, utilisant un kit ${ }^{3} \mathrm{H}$ RIA de bioMérieux (Charbonnières-les-Bains, France). La FSH, la LH et la SHBG étaient dosées par tests immunoradiométriques (Coatria, bioMérieux, France). La T biodisponible était mesurée selon la méthode pré- 
cédemment décrite [15]. Brièvement, le test était basé sur la séparation de la $\mathrm{T}$ biodisponible par une précipitation spécifique de la SHBG avec du sulfate d'ammonium (50\% saturation) après équilibre de l'échantillon de sérum avec une dose de traceur de testostérone tritiée $\left(\left[1,2,6,7-{ }^{3} \mathrm{H}\right]\right.$ testostérone) à $37^{\circ} \mathrm{C}$.

\section{Analyse statistique.}

Les résultats sont exprimés en moyennes. \pm DS (déviation standard). Le test non paramétrique de Mann-Withney a été utilisé pour la comparaison de différents paramètres.

\section{RESULTATS}

Parmi les 40 sujets atteints de dysfonction érectile, 13 avaient une étiologie psychogénique, 7 une étiologie vasculogénique, (5 artériopathies et 2 fuites caverneuses), et 8 avaient une étiologie iatrogénique ( $\beta$-bloqueurs, inhibiteurs de l'enzyme de conversion, diurétiques), et aucune étiologie n'était trouvée chez 12 patients. Les dysfonctions érectiles étaient plus fréquentes chez les sujets les plus âgés (37/40 avaient plus de 35 ans) que chez les sujets les plus jeunes ( $3 / 40$ avaient moins de 35 ans).
Les résultats des tests hormonaux sont donnés dans le tableau 1. Dans le but d'étudier l'effet de l'âge sur la fonction gonadique, nous avons comparé le groupe contrôle le plus jeune ( $<35$ ans) au groupe contrôle le plus âgé ( $>40$ ans). La testostérone totale n'était pas significativement différente dans les deux groupes. Toutefois un hypoandrogénisme se révèle chez les sujets les plus âgés puisque la $\mathrm{T}$ biodisponible sérique était significativement plus basse dans ce groupe $(p<0,01)$ que dans le groupe de sujets jeunes sains. Cette diminution était associée à une augmentation significative de la SHBG sérique dans le groupe des sujets âgés sains. La FSH, la LH et l'oestradiol sériques n'étaient pas significativement différents dans les deux groupes contrôles.

La testostérone totale n'était pas significativement différente dans les groupes d'étiologies psychogéniques, vasculogéniques, iatrogéniques ou d'étiologies inconnues quand ils étaient comparés au groupe contrôle de même âge. Pour ce même paramètre, les différences entre les sousgroupes d'étiologies différentes et comparés deux à deux n'était pas significatives.

La T biodisponible sérique était significativement plus basse dans les étiologies psychogéniques $(p<0,05)$ et dans les étiologies

Tableau 1 : Androgènes, T biodisponible, SHBG, oestradiol sériques (moyenne \pm SD), hormone lutéinique et stimulante folliculaire de patients atteints de dysfonction érectile.

\begin{tabular}{|c|c|c|c|c|c|c|c|}
\hline Groupe & $\underset{(\text { moyenne }}{\text { Age }} \pm$ DS $)$ & $\begin{array}{l}\text { Testosterone } \\
(\mathrm{nmol} / \mathrm{l})\end{array}$ & $\begin{array}{c}\text { T biodisponible } \\
(\text { nmol/l) }\end{array}$ & $\begin{array}{c}\text { SHBG } \\
(\mathbf{n m o l} / \mathbf{l})\end{array}$ & $\begin{array}{l}\text { Oestradiol } \\
(\mathrm{pmol} / \mathrm{l})\end{array}$ & $\underset{(\mathbf{m U I} / \mathbf{l})}{\text { FSH }}$ & $\underset{(\mathbf{m U I} / \mathbf{l})}{\mathbf{L H}}$ \\
\hline Controle $n^{\circ} 1(n=10)$ & $28 \pm 3$ & $22.7 \pm 2.8$ & $10.9 \pm 2.8$ & $22.4 \pm 4.3$ & $129.2 \pm 27.3$ & $4.7 \pm 3.1$ & $5.3 \pm 2.4$ \\
\hline Controle $n^{\circ} 2(n=9)$ & $52 \pm 8$ & $22.3 \pm 4.4$ & $7.5 \pm 2.3 \bullet \bullet$ & $31.5 \pm 6.2 \bullet \bullet$ & $125.7 \pm 24.9$ & $5.9 \pm 2.3$ & $5.4 \pm 2.5$ \\
\hline Psychogénique (n=13) & $51 \pm 7$ & $18 \pm 6$ & $5.4 \pm 1.3^{*}$ & $24 \pm 10^{*} \Delta \Delta$ & $130.4 \pm 51$ & $4 \pm 4.3^{*}$ & $3.8 \pm 2.5^{*}$ \\
\hline Vasculogénique $(n=7)$ & $49.5 \pm 11$ & $25.3 \pm 10.8$ & $5.4 \pm 1.8$ & $45.5 \pm 18$ & $169.1 \pm 72.6$ & $5.4 \pm 3.8$ & $5.4 \pm 2.2$ \\
\hline Iatrogénique (n = 8) & $57 \pm 7$ & $18.9 \pm 8.5$ & $5.9 \pm 4.1$ & $28.1 \pm 14.3$ & $98.1 \pm 27$ & $5.1 \pm 3.3$ & $5.2 \pm 2.5$ \\
\hline Idopathique (n = 12) & $48 \pm 10$ & $20.9 \pm 6$ & $5.44 \pm 1.4^{*}$ & $33.8 \pm 12$ & $123+48$ & $4.2 \pm 2.1$ & $5.9 \pm 2.8$ \\
\hline
\end{tabular}


idiopathiques $(\mathrm{p}<0,05)$ en comparaison au groupe contrôle de même âge. Pour ce même paramètre, les différences entre les sous-groupes d'étiologies différentes et comparés deux à deux n'était pas significatives. De plus, dans les étiologies psychogéniques, la FSH et la LH étaient significativement plus basses que dans le groupe contrôle de même âge $(\mathrm{p}<0,05)$.

La SHBG sérique était, par rapport au groupe contrôle, significativement plus élevée dans les étiologies psychogéniques $(\mathrm{p}<$ 0,05 ) et plus élevée en moyenne, mais non significativement dans les étiologies vasculaires $(p<0,05)$. Pour ce paramètre, aucune différence significative n'était trouvée entre les groupes de patients d'étiologies iatrogéniques ou d'étiologies idiopathiques et le groupe contrôle de même âge.

La SHBG était significativement plus élevée dans le groupe de patients d'étiologies vasculaires que dans le groupe de patient d'étiologies psychogéniques.

\section{DISCUSSION}

Nous avons étudié le statut hormonal de patients atteints de dysfonction érectile de différentes étiologies : psychogénique, vasculogénique, iatrogénique et étiologique et selon l'âge, dans deux groupes contrôles différents.

Aucune baisse significative de la testostérone totale dans tous les sous-groupes n'a été observée. Par contre, la concentration sérique de SHBG augmente avec l'âge et dans le groupe d'étiologie psychogénique. Aucune différence significative n'apparaissait pour tous les paramètres étudiés entre le groupe d'étiologie iatrogène et le groupe contrôle.

Une diminution de la $\mathrm{T}$ biodisponible, associée à une testostérone totale normale dans le sérum, a été précédemment décrite chez des sujets âgés (Nankin et al., 1986). D'autres auteurs ont trouvé que la baisse de la testostérone totale devenait plus évidente avec l'avancée en âge, mais de façon moins significative que la $\mathrm{T}$ biodisponible. De plus, la baisse de la $\mathrm{T}$ biodisponible apparaissait plus précocement que celle de la testostérone totale [18].

Des résultats similaires ont été trouvés chez l'homme âgé avec une baisse significative de la $\mathrm{T}$ biodisponible et totale [13]. L'âge est le paramètre prédictif le plus important de la concentration en SHBG, qui augmente parallèlement [14]. De plus la concentration de SHBG est négativement corrélée avec la concentration de testostérone libre et de T liée à l'albumine [14]. Dans notre étude, nous n'avons pas mis en évidence de diminution de la T totale chez les sujets âgés comme certains auteurs l'ont rapporté [16 et 17].

Ce résultat pourrait être lié à l'âge moyen peu élevé du groupe de sujets âgés $(52 \pm 8$ ans) et du fait que la baisse de la $\mathrm{T}$ totale survient plus tardivement.

De façon étonnante, nous avons trouvé que la $\mathrm{T}$ biodisponible baisse de façon significative lors de dysfonction érectile d'étiologie inconnue en comparaison au groupe contrôle de même âge, et sans aucune variation de la SHBG. Nous n'avons pas trouvé un tel résultat dans la littérature.

Plusieurs explications sont possibles. Tout d'abord, ce groupe pourrait comporter plusieurs sujets atteints de dysfonction organique indétectable à l'examen clinique et aux examens complémentaires, chez lesquels une baisse de la $\mathrm{T}$ biodisponible pourrait survenir avant l'apparition de symptôme d'une défaillance organique. La testostérone biodisponible serait ainsi un test hormonologique plus sensible que la T totale dans l'exploration du caractère organique d'une dysfonction érectile. Ces patients nécessiteraient une surveillance prospective clinique et biologique régulière et prolongée dans le but de mettre en évidence la survenue d'une étiologie organique à la dys- 
fonction érectile. En second lieu, il serait possible qu'un hypogonadisme se manifeste uniquement par une baisse de la $\mathrm{T}$ biodisponible. Les mécanismes de régulation de la SHBG pourraient intervenir plus tardivement quand la testostérone totale décroît [2]. Ce résultat montre que le taux de la $\mathrm{T}$ biodisponible serait plus sensible que celui de la $\mathrm{T}$ totale pour détecter un hypogonadisme.

L'augmentation de la SHBG, mais de façon non significative ici, trouvée dans le groupe d'étiologie vasculogénique, a néanmoins été mise en évidence par certains auteurs et de façon significative [23]. Une explication possible de cette différence de résultats est que notre groupe de patients n'était pas suffisamment nombreux pour obtenir une différence statistiquement significative. Ce groupe est hétérogène et il serait ainsi intéressant d'étudier deux groupes séparés suffisamment nombreux, l'un regroupant les patients atteints de dysfonction érectile associée à une fuite caverneuse, l'autre regroupant les patients atteints d'artériopathie.

Nous avons observé une diminution de la concentration de $\mathrm{T}$ biodisponible et une augmentation de celle de la SHBG des patients atteints de troubles d'origine psychogène, associées à une diminution de la FSH et de la LH.

Une augmentation significative de la concentration de la SHBG a été décrite dans les dysfonctions érectiles d'étiologie psychogénique [23]. Par contre, nous n'avons pas, à notre connaissance, retrouvé dans la littérature une baisse significative da la FSH et de la LH chez les patients atteints de dysfonction érectile d'origine psychogénique. Dans notre étude, un certain nombre de patients de ce sous-groupe avaient un syndrome dépressif. Des perturbations des fonctions hypothalamo-hypophysaires ont été décrites chez ces sujets, en particulier l'atteinte de l'axe corticotrope. Il serait possible que ces dysfonctions hypothalamo-hypophysaires atteignent l'axe gonadique. Ceci reste à confirmer par une étude complémentaire chez des sujets dépressifs atteints de dysfonctions érectiles.

Une autre hypothèse possible est que les sujets atteints d'une dysfonction érectile d'origine psychogène, en particulier de dépression, ont une baisse de la libido et donc une activité sexuelle réduite. Or, la testostérone totale augmente avec l'activité sexuelle et diminue avec la baisse de l'activité. Cet effet régulateur pourrait s'observer précocement par la baisse de la testostérone biodisponible ou $\mathrm{T}$ biodisponible.

En conclusion, nous avons étudié le statut androgénique de patients atteints de dysfonction érectile d'origines différentes : vasculaire, psychogène, iatrogène et d'étiologie idiopathique.

Nous avons montré que certaines étiologies organiques étaient associées à un hypoandrogénisme du fait d'une augmentation de la SHBG liant avec une forte affinité la testostérone. Le statut androgénique est normal dans les dysfonctions érectiles d'origine iatrogénique. Au contraire, dans le groupe de patients atteints de dysfonction érectile d'origine psychogénique, la concentration de T biodisponible, la SHBG augmente, et ces modifications sont associées à une diminution significative de la FSH et de LH.

Le principal résultat de cette étude est que dans un groupe de patients atteints de dysfonction érectile d'origine idiopathique, la concentration de SHBG-NT est diminuée par rapport au groupe témoin. Ainsi, il apparaît un hypogonadisme périphérique sans mise évidence de défaillance organique. Une étude prospective pourrait montrer si une étiologie indétectable au moment de l'étude se révèle ultérieurement. De plus, les résultats de cette étude justifient une thérapeutique androgénique chez les patients atteints de dysfonction érectile associée à une diminution de la $\mathrm{T}$ biodisponible. 


\section{REFERENCES}

1. AASEBO U., GYLTNESS A., BREMNES R.M., AAKVAAG A., SLORDAL L. : Reversal of sexual impotence in male patients with chronic obstructive pulmonary disease and hypoxemia with longterm oxygen therapy. Journal of Steroid Biochemistry \& Molecular Biology, 1993, 46 : 799-803.

2. ANDERSON D.C., BARTSCH W., BECKER H., PINKENBURG F. A., KRIEG M. : Hormone blood levels and their inter-relationships in normal men and men with benign prostatic hyperplasia (BPH). Acta Endocrinologica, 1979, 90 : 727-736.

3. BAKER H.W., BURGER H.G., de KRESTER, DULMANIS A., HUDSON B., O'CONNOR S. et al. : A study of endocrine manifestations of hepatic cirrhosis. Quartely Journal of Medecine, 1976, 45: 145-178.

4. CUNDY T., BOMFORD A., BUTLER J., WHEELER M., WILLIAMS R. : Hypogonadism and sexual dysfonction in hemochromatosis : the effect of cirrhosis and diabetes. Journal of clinical Endocrinology \& Metabolism,1989, $69:$ 110-116.

5. DAVIDSON J.M., CHEN J.J., CRAPO L., GRAY G.D., GREENLEAF W.J., CATANIA J.A. : Hormonal change and sexual function in aging men. Journal of Clinical Endocrinology \& Metabolism, 1983,57 : 71-77.

6. DESLYPERE J.P., VERMEULEN A. : Leydig cell function in normal men: effect of age, life-style, residence, diet, and activity. Journal of Clinical Endocrinology \& Metabolism, 1987, 59 : 955-962.

7. FARNSWORTH W.E., CAVANAUGH A.H., BROWN J.R., ALVAREZ I., LEWANDOWSKI L.M. : Factors underlying infertility in the alcoholic. Archives of Andrology, 1978, $1: 193-195$.

8. FARTHING M.J., REES L.H., EDWARDS C.R., DAWSON A.M. : Male gonadal function in coeliac disease : 2. Sex hormones. Gut, 1983, 24 : 127-135.

9. FREIDMAN D.E., CLARE A.D., LESLEY H.R., GROSSMAN A. : Should impotent males who have no clinical evidence of hypogonadism have routine endocrine sceerning ? The Lancet, 1986; May $3: 104$.

10. GEISTHOVEL W., NIEDRFGERKE U., MORGNER K.D., WILLMS B., MITZKAT H.J. : Androgen status of male diabetics. Total testostérone before and folowing stimulation with HCG, free testostérone, and testostérone binding capacity of patients with and without potency disorders. Medizinische Klinik-Munich, 1975, 36 : 1417-1423.

11. GEISTHOVEL W., VON ZUR MUHLEN A. : Testostérone-binding capacity, free-plama testostérone concentration in andrological patients. Deutsche Medizinische Wochenschrift, 1976, $2: 37-42$.
12. KLEY H.K., STREMMEL W., KLEY J.B., SCHLAGHECKE R. : Testostérone treatment of men with idiopathic hemochromatosis. Clinical Investigator, 1992, $70: 566-572$.

13. KORENMAN S.G., MORLEY J.E., MOORADIAN A.D., DAVIS S.S., KAISER F.E., SILVER A.J. et al. : Secondary hypogonadism in older men : its relation to impotence. Journal of clinical Endocrinology \& Metabolism, 1990, 71 : 963968.

14. LONGCOPE C., GOLFIELD S.R.W., BRAMBILLA D.J., MCKINLAY J.M. : Androgens, Estrogens, and sex hormone-binding globulin in middle-aged men. Journal of Clinical Endocrinology \& Metabolism, $1990,71: 1442-1446$.

15. LORIC S., GUECHOT J., DURON F., AUBERT P., GIBOUDEAU J. : Non sex-hormone-binding globulin-bound testostérone determination in serum and its diagnostic value in hirsute women. Clinical Chemestry, 1988, 34 : 329-339.

16. MORLEY J.E., KAISER F.E. : Sexual function with advancing age. Medical Clinics of North America, 1989, $73: 1483-1495$.

17. NAHOUL K., ROGER M. : Age-related decline of plasma bioavailable testostérone in adult men. Journal of Steroid Biochemistry, 1990, 35 : 293299.

18. NANKIN H.R., CALKINS J.H. : Decreased bioavailable testostérone in aging normal and impotent men. Journal of clinical Endocrinology \& Metabolism, 1986, 63 : 1418-1420.

19. RUBENS R., DHONT M., VERMEULEN A. : Further studies on leydig cell function in old age. Journal of clinical Endocrinology \& Metabolism, $1974,39: 40-45$.

20. SELBY C. : Sex hormone binding globuline: origin, function and clinical significance. Ann Clin Biochem, 1990, $27: 532-541$.

21. STEARNS E.L., MACDONNELL J.A., KAUFMAN B.J., PADUA R., LUCMAN T.S., WINTER J.S. and al.: Declining testicular function with age. Am. J. Med, 1974, 74 : 792-735.

22. VERMEULEN A., DESLYPERE J.P. : Testicular endocrine function in ageing male. Maturitas, 1985, $7: 273-279$.

23. ZIAJKA I., HECKLER M., MUSQUAR M., BERTRAND F., NABET F., DAP G. et al. : Dosage de la sex-hormone binding-globulin (SHBG) dans le bilan d'impuissance masculine. Résultats préliminaires. Biologie prospective. Comptes rendus du $7 \mathrm{e}$ colloque de Pont-à-Mousson, 1989, 443-446. 


\section{ABSTRACT \\ J. Devignes, W. Mahmoud, J. Guechot, G. ARVIS ET J. GiboUdEAU}

Is serum non-sex hormone binding globulin-bound (non-SHBG-bound) testosterone more sensitive than total testosterone $(T)$ in men presenting érectile dysfonction ? Non-SHBG-bound testosterone level has been shown to undergo decrease whereas SHBG level increases in middle-aged men without érectile dysfonction. Serum SHBG increase has been found in secondary organic etiology of érectile dysfonction. The aim of this work was to study hormonal status in men presenting érectile dysfonction. Serum SHBG, T, bioavailable $T$, luteinizing hormone (LH) and folliculin stimulating hormone (FSH) levels were measured in 40 men presenting érectile dysfonction. They were divided into four groups according to their etiology : psychogenic, vasculogenic, iatrogenic, and unknown etiologies. In order to consider the effect of the age, each group was compared with age-related healthy controls without any érectile dysfonction. Non-SHBG-bound-T decreased with age and SHBG increased, while serum $T$ was similar in young and elderly control subjects. In the vasculogenic subjects, SHBG was higher than in the controls, but not significantly. In the patients with érectile dysfonction of unknown etiologies, non-SHBGbound-T was lower than in the controls without increase of SHBG. In the psychogenic patients, SHBG was higher than in the controls while total $T$ was similar in both groups. This study allowed to investigate androgen status of men suffering of érectile dysfonction according to their etiology. The following step would be to study the rate of success of appropriate hormonal therapy in patients in which peripheral hypogonadism occurs.

Keywords : érectile dysfonction, hypogonadism, bioavailable testosterone, sex-hormone-binding globulin. 